\title{
Fossil Psychodoid Flies and Their Relation to Parasitic Diseases
}

\author{
Dany Azar ${ }^{+}$, André Nel* \\ Department of Biology, Faculty of Sciences II, Lebanese University, B.P. 90356, Jdeibeth-el-Matn, Lebanon and Saint-Joseph \\ University, Campus of Sciences and Technology, Mar Roukos (Mkalles), B.P. 11-1514 Beirut, Lebanon \\ *Laboratoire d'Entomologie, Muséum National d'Histoire Naturelle, Paris, France
}

\begin{abstract}
Psychodid sand flies are blood-sucking fly vectors of several parasitic diseases. The oldest definitive record of this group is from the Lower Cretaceous amber of Lebanon (circa-135 to-125 My), but the high diversity within this group supports the idea that the psychodoids originated much earlier in history. The palaeontology demonstrates that the Lower Cretaceous representatives of the different subfamilies of Psychodidae had similar morphology and were blood-feeders, which supports Hennig's hypothesis on the ground plan structure of this family. Historical relationship between sand flies and diseases is unclear up to the present time, but this relationship could be as old as the origin of psychodoids because of the blood-feeding life mode.
\end{abstract}

Key words: fossil psychodoidea - blood-feeder - sand flies - amber

Phlebotomine sand flies (Phlebotominae) are a group of Diptera with blood-sucking adaptations. They are either considered as belonging to the family Psychodidae, or a genuine family Phlebotomidae forming with the Psychodidae the superfamily of Psychodoidea (Rohdendorf 1964, 1974, Lewis 1973, Williams 1993). Sand flies are characterized by their densely hairy wings, giving them a moth-like appearance. Phlebotomines are distinguished from other members of the family, if we consider them as a subfamily within Psychodidae, by the way they hold their wings erected above the body in a vertical $\mathrm{V}$, whereas members of other psychodid subfamilies held their wings flat and near the body. They occur in a wide range of habitats. The different taxa often have very specific habitat requirements. Sand flies are characteristically encountered in the Old World in dry, semi-arid, and temperate areas, breeding in leaf litter or moist fissures in the soil, whereas in the New World, they occur mainly in tropical forests and savannas.

\section{LIFE CYCLE AND BIOLOGY OF SAND FLIES}

It is difficult to study the life cycle of sand flies and their relation to diseases because the larvae are tiny and their habitats are not well known. Adults are also often hard to find, and larvae usually impossible (Lewis 1973). The study in the laboratory of these arthropod vectors is also more difficult than for others. The entire life cycle takes 20-40 days except in diapausing species, i.e. those that stop developing when conditions become too cold.

The females lay 30-70 eggs by scattering them around a potential breeding site. They hatch within 1-2 weeks. Larvae inhabit and feed on dead organic matter. They are found in damp places containing organic matter such as cracks in walls or rocks, animal burrows and shelters, caves, termite hills, tree holes or in leaf litter. In regions

${ }^{+}$Corresponding author. Fax: +33(0)1-40-79-36.99. E-mail: azar@mnhn.fr

Received 26 August 2002

Accepted 25 November 2002 with cool winters, larvae diapause in the fourth (final) instar. Pupal development takes 5-10 days. Adults emerge from the pupae in darkness, often just before dawn. Only the female sucks blood, this food being used for egg production. Both males and females feed on sugary secretions from plants or from honeydew produced by homopteran bugs. Mating takes place at or near hosts: the males congregate in leks, on or near the host. They produce sex pheromones. Females home in on hosts using both host odour and the odour produced by the males. Male wing vibration can be important in encouraging females to mate. Seasonal activity of adults is mainly affected by temperature and rainfall. Adults are mainly active in the early morning, evening, and at night, although they can bite during the day if disturbed. When inactive, adult sand flies live in resting sites that are characteristic of each species. Resting sites are often similar or near to the larval breeding sites, usually cool, humid and dark places. Sand flies are able to survive in dry environments by withdrawing to cool, humid, resting sites during the day and then becoming active at night when ambient temperatures drop and humidity increases.

Sand flies are widely distributed in the tropics and other warm mainland areas, and extend northwards to latitudes in the region of $50^{\circ} \mathrm{N}$, such as $48^{\circ}$ in the CIS (ex Soviet Union), Savignie near Beauvais in France, Jersey in the English Channel, and Kamloops in Canada (Lewis 1973). The habit of breeding in soil makes sand flies independent of surface water, their small size enables them to use microclimates, and their nocturnal habits help them to avoid heat. Therefore, these insects inhabit wide areas despite their delicate structure and rather limited flight range.

\section{RELATION TO DISEASES}

There are about 700 species of phlebotomine sand flies. About 70 of them are important vectors considered to transmit diseases to human, including protozoan parasites (Leishmania) (Adler \& Theodor 1957), bacteria (Bartonella bacilliformis), or viruses (Vesicular Stomatisis Virus, Phlebovirus Genus, Sandfly Fever Virus, Rift Valley Fever Virus, Toscana Virus, Chagres Virus, Punta Toro Virus). 
The diseases of the 'Leishmaniasis' group are caused by intracellular protozoan parasites of the genus Leishmania. There is a large number of species and subspecies of Leishmania, grouped according to their development within the sand fly vector. Among the 30 known species, 21 infect humans. All are spread by bites of infected sand flies. Leishmania is named after Dr Leishman, who first described it in London in May 1903. Leishmania are intracellular parasites that infect the mononuclear phagocytes. The spectrum of human disease ranges from self-healing localized ulcers to widely disseminated progressive lesions of the skin, mucus membranes, even the entire reticuloendothelial system. There are two major forms of leishmaniases: cutaneous and visceral leishmaniases. Cutaneous leishmaniases afflict 1.5 millions of people all over the World. They cause sores on the skin, which can be disfiguring. They can spread to mucus linings of the nose and throat to cause a less common disease known as mucosal leishmaniasis. In contrast, visceral leishmaniases cause a disruption of the immune system, resulting in fever, enlargement of the liver, enlargement of the spleen, anemia, and loss of weight. There are half a million new cases of visceral leishmaniases around World each year. If untreated, visceral leishmaniases are fatal.

Some sand flies are also vectors of several animal trypanosomes (Wallace \& Hertig 1968) and probably of an Endotrypanum of sloths (Shaw 1964). A reptilian malaria parasite has been found to develop to the sporozoite stage in two species of Lutzomyia (Ayala \& Lee 1970).

Bartonella bacilliformis is bacterium causing the disease bartonellosis, which takes the form of the dermal Verruga Peruana or the Severe Oroya Fever or Carrion's Disease, in Northwestern part of South America (Peru, Colombia and Ecuador) (Lewis 1973).

Vesicular Stomatitis is an acute viral vesicular disease that primarily affects cattle, horses, and swines. The virus that causes Vesicular Stomatitis has a wide host range. This disease also occasionally affects sheep and goats. Many species of wild animals, including cattle, deers, bobcats, pigs, goats, racoons, and monkeys, have been found to be susceptible hosts. Humans can also become infected with Vesicular Stomatitis when handling affected animals. The etiologic agent, Vesicular Stomatitis Virus (VSV), is a rhabdovirus. One type of VSV is spread by phlebotomine sand flies. Once introduced into a herd, the disease apparently moves from animal to animal by contact or exposure to saliva or fluid from ruptured lesions.

The genus Phlebovirus comprises over 50 viruses which are transmitted by mosquitoes or phlebotomine flies. Rift Valley Fever Virus and Sand Fly Fever Virus are the most medically important agents which have been the greatest focus of study. Toscana Virus occurs in the Northern and Western Mediterranean area. Chagres and Punta Toro Viruses occur in the New World.

\section{IMPORTANCE OF PHYLOGENETIC ANALYSIS AND FOSSIL STUDY}

An understanding of evolutionary relationships among different subfamilies and species of psychodoid flies and especially phlebotomine sand flies is of epide- miological importance, because it helps to predict the transmission patterns of different species and strains of organisms causing diseases to the humans. Therefore a study of the fossil psychodoid flies is of great importance for highlighting the real evolution and phylogeny of this group. Azar et al. (1999) demonstrated that the addition of fossil psychodoid flies to the phylogeny based on recent taxa does not disturb its topology, but could be a test of the historical value of the phylogeny through the character homoplasy. Moreover, the addition of the fossil taxa demonstrates that the phylogenetical history of Psychodoidea is clearly more complicated than suggested by the study of the recent taxa alone.

The family Psychodidae is well represented in the dipteran fossil record: 18 genera and about 52 fossil species were described before 1994 (Evenhuis 1994). The knowledge of fossil taxa has greatly improved up to least 30 genera and more than 75 species since the works of Ansorge (1994), Azar et al. (1999), Azar and Nel (2002), Nel et al. (2002).

Lambrecht (1980) estimated that the psychodoids originated as long ago as the Permian/Triassic boundary. Even if the oldest fossil record of psychodoid is from the Triassic of North America and some Jurassic fossils are attributed to this lineage (Kalugina \& Kovalev 1985, Fraser et al. 1996), the oldest genuine sand flies are from the Lower Cretaceous Lebanese amber (Hennig 1972). About 20 fossil psychodoid taxa were recently discovered in the Lebanese amber (nine Psychodinae, six Phlebotominae, and one possible Trichomyinae). This suggests that all the recent subfamilies of psychodoids were already present at this time. Also, one Trichomyinae and two Psychodinae are recorded from the Upper Cretaceous Taimyr amber, one Trichomyinae from the Upper Cretaceous Canadian amber; one Trichomyinae from the Upper Cretaceous New Jersey amber; and one Psychodinae from the Lower Cretaceous lacustrine Santana formation in Brazil. Lastly, a new genus of uncertain subfamilial affinities is recorded from the Lebanese, Spanish (Alava) and French (Charentes-Maritimes) ambers. All these discoveries suggest that the psychodoid flies were already well diversified by the Cretaceous time. In addition, most of the psychodoids flies from the Lower Cretaceous were probably of the blood-feeder type. This supports the theory of Hennig (1972) about the hypothetical phlebotomine-like ancestor of the Psychodidae. There is a 'gradation' from a well developed, blood-sucking, phlebotomine-like type to a reduced, probably pollen-eating and psychodine-like type of mouth parts (Figure). These fossils also show a 'gradation' from five to four palpomeres: some fossil Psychodinae have a faint furrow in one of their palpomeres, which could be the remain of the fusion of two palpomeres. These fossils also illustrate a 'gradual' development of the eye bridge in psychodoid flies. Furthermore, some of these Cretaceous Psychodinae have a well developed male phlebotomid-like genital apparatus. The Lebanese Lower Cretaceous phlebotomines have a small remaining part of the anal vein unlike the more or less completely reduced anal vein of the recent taxa. 


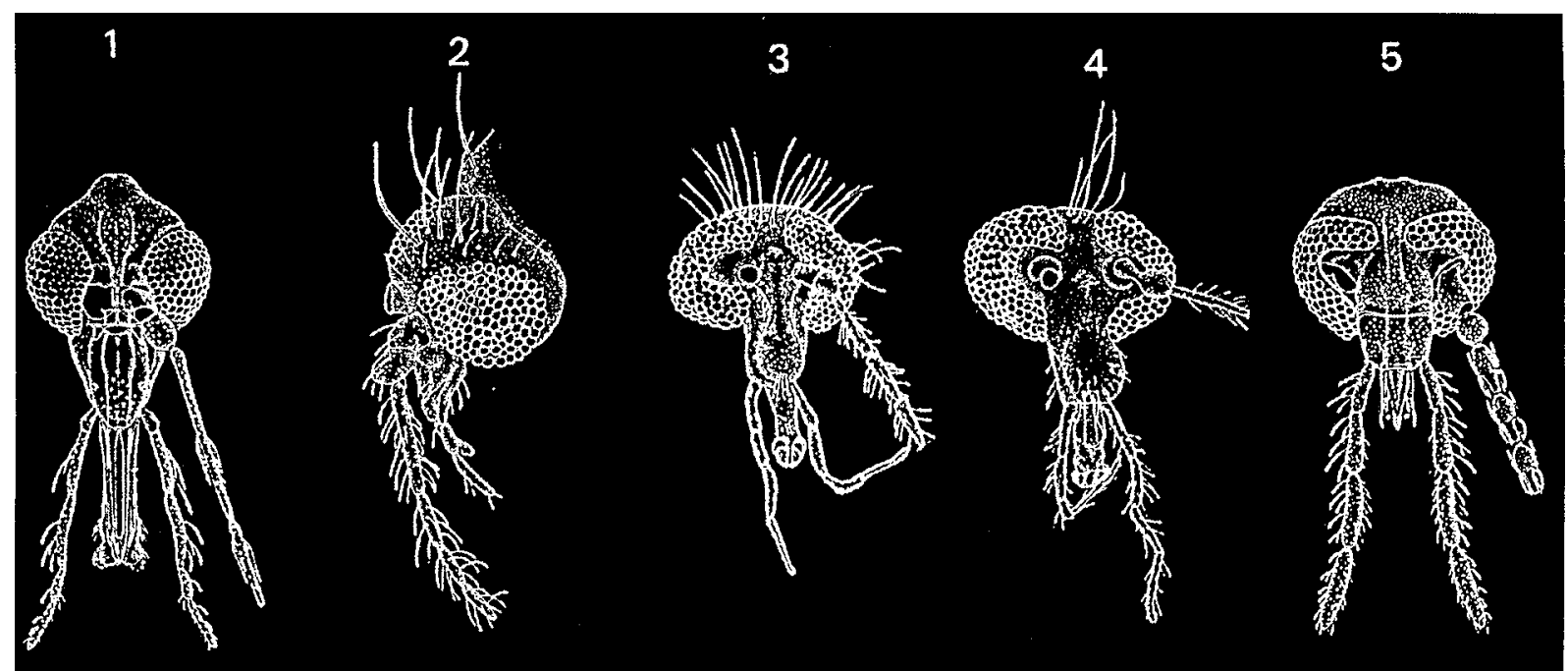

Evolutive gradation on three structures in Pschodoidea: form of eyes, mouth parts and maxillary palpes. 1: Lutzomyia sp. (Recent Phlebotominae); 2: Protopsychoda nadiae Azar et al. 1999 (fossil Psychodinae from Lebanese amber); 3: Paleopsychoda jacquelinea Azar et al. 1999 (fossil Psychodinae from Lebanese amber); 4: Paleopsychoda solignaci Azar et al. 1999 (fossil Psychodinae from Lebanese amber); 5: Psychoda cinerea (Recent Psychodinae). In Recent Phlebotominae, the eyes are rounded. In Psychodinae, the eyes form an eye-bridge, the Lower Cretaceous fossils show several situations between the two types of eyes. Phlebotominae are hematophagous whereas Psychodinae are polliniphagous. The fossils of Psychodinae from the lower Cretaceous have mouth-parts of phlebotominetype, with five maxillary palps in Phlebotominae, instead of four in Psychodinae. The fossil Psychodinae have four maxillary palps with a constriction on one of the palpomeres, which may result from a fusion of two palpomeres.

Historical relationships between psychodoids and parasitic diseases remain unclear to present time. Over the years, parasitic organisms such as nematods, protozoa and viruses have become associated with sand flies. In the present time some species of sand flies feed on different vertebrates including humans and reptiles; these latter are cold-blooded vertebrates. It is possible that similar associations occurred very early in time between some of the psychodoids and contemporaneous vertebrates like mammals, dinosaurs, birds or pterosaurs, etc. Even, new parasites transmitted by psychodoid flies could have contributed to the extinction of dinosaurs by the end of Cretaceous period. Relationships between psychodoids and parasitic diseases are as old as the origin of the first ones, due to their blood-feeding system.

\section{REFERENCES}

Adler S, Theodor O 1957. Transmission of disease agents by phlebotomine sandflies. Ann Rev Entomol 2: 203-226.

Ansorge J 1994. Tanyderidae and Psychodidae (Insecta: Diptera) from the Lower Jurassic of Northeastern Germany. Paläontol Zeit 68: 199-210.

Ayala SC, Lee D 1970. Saurian malaria: development of sorozoites in two species of phlebotomine sand flies. Science 167: 891-892.

Azar D, Nel A 2002. New Cretaceous psychodid flies from Lebanese amber and Chapada do Araripe, Santana, formation (Brazil) (Insecta, Diptera, Psychodidae). Ann Soc Entomol Fr (in press).

Azar D, Nel A, Solignac M, Paicheler J-C, Bouchet F 1999. New genera and species of phlebotomid and psychodid flies from the Lower Cretaceous amber of Lebanon (Insecta: Diptera: Phlebotomidae, Psychodidae). Palaeontol 42: 1131-1136.

Evenhius NL 1994. Catalogue of the Fossil Flies of the World
(Insecta:Diptera), Backbugs, Leiden, 568 pp.

Fraser NC, Grimaldi DA, Olsen PE, Axsmith B 1996. A Triassic Lagerstätte from Eastern North America. Nature 380: 615-619.

Hennig W 1972. Insektenfossilien aus der unteren Kreide. IV. Psychodidae (Phlebotominae), mit einer kritischen Übersicht über das phylogenetische System der familie und die bisher beschriebenen Fossilien (Diptera). Stutt Beit Natur (B) 241:1-69.

Kalugina NS, Kovalev VG 1985. [Dipterous Insects of Jurassic Siberia], Paleontol Inst Akad Nauk, Moscow, 198 pp. [In Russian].

Lambrecht FL 1980. Palaeoecology of tsetse flies and sleeping sickness in Africa. Proc Am Philos Soc 124: 367-85.

Lewis DJ 1973. Phlebotomidae and Psychodidae (Sand-flies and Moth-flies). Chapter 3c. in KGV Smith, Insects and Other Arthropods of Medical Importance, British Museum of Natural History, London, p. 155-179.

Nel A, Menier J-J, De Ploëg G 2002. The oldest representative of the Trichomyiinae from the Lowermost Eocene amber of the Paris Basin (France) (Diptera: Psychodidae). Ann Soc Entomol Fr (in press).

Rohdendorf BB 1964. [Historical development of dipterous insects]. Trudy Palaeontol Inst Akad Nauk SSSR 100: 1312, [In Russian].

Rohdendorf BB 1974. The Historical Development of Diptera. Translated from the Russian by JE Moore \& I Theile. The University of Alberta Press, Edmonton [English translation of Rohdendorf 1964].

Shaw JJ 1964. A probable vector of Endotrypanum schaudini in the sloth Choloepus hoffmani, in Panama. Nature 201: 417-418.

Wallace FG, Hertig M 1968. Ultrastructural comparison of promastigote flagellates (leptomonads) of wild-caught Panamanian Phlebotomus. J Parasitol 54: 606-612.

Williams P 1993. Relationships of phlebotomine sand flies (Diptera). Mem Inst Oswaldo Cruz 88: 177-183. 